\title{
Intratester Reliability and Construct Validity of a Hip Abductor Eccentric Strength Test
}

\author{
Richard A. Brindle, David Ebaugh, and Clare E. Milner
}

\begin{abstract}
Context: Side-lying hip abductor strength tests are commonly used to evaluate muscle strength. In a "break" test, the tester applies sufficient force to lower the limb to the table while the patient resists. The peak force is postulated to occur while the leg is lowering, thus representing the participant's eccentric muscle strength. However, it is unclear whether peak force occurs before or after the leg begins to lower. Objectives: To determine intrarater reliability and construct validity of a hip abductor eccentric strength test. Design: Intrarater reliability and construct validity study. Participants: Twenty healthy adults (26 [6] y; 1.66 [0.06] m; 62.2 [8.0] kg) made 2 visits to the laboratory at least 1 week apart. Main Outcome Measures: During the hip abductor eccentric strength test, a handheld dynamometer recorded peak force and time to peak force, and limb position was recorded via a motion capture system. Intrarater reliability was determined using intraclass correlation, SEM, and minimal detectable difference. Construct validity was assessed by determining if peak force occurred after the start of the lowering phase using a 1-sample $t$ test. Results: The hip abductor eccentric strength test had substantial intrarater reliability (intraclass correlation $_{(3,3)}=.88 ; 95 \%$ confidence interval, .65-.95), SEM of $0.9 \% \mathrm{BWh}$, and a minimal detectable difference of $2.5 \% \mathrm{BWh}$. Construct validity was established as peak force occurred $2.1(0.6)$ seconds (range: 0.7-3.7 s) after the start of the lowering phase of the test $(P \leq .001)$. Conclusion: The hip abductor eccentric strength test is a valid and reliable measure of eccentric muscle strength. This test may be used clinically to assess changes in eccentric muscle strength over time.
\end{abstract}

Keywords: peak force, break test, peak torque

Weakness of hip abductor muscles during eccentric loading and associated medial collapse have been hypothesized as an etiology of overuse and noncontact injuries, such as patellofemoral pain syndrome ${ }^{1}$ and anterior cruciate ligament tears. ${ }^{2}$ Furthermore, hip abductor muscle eccentric strengthening is a common component of treatment. ${ }^{3}$ In a side-lying hip abductor strength test, an adduction force is applied to the patient's limb through a handheld dynamometer that either matches or exceeds the patient's maximum isometric force. In a hip abductor eccentric strength test or "break test," the tester exceeds the patient's maximum isometric force and pushes the limb down. ${ }^{4-6}$ The peak force recorded by the handheld dynamometer is assumed to be generated while the leg is being lowered (the lowering phase). However, the time of the peak force during this test has not been established. Thus, it is unclear whether the peak force during a hip abductor eccentric strength test occurs before or after the leg begins to lower.

For measures to be useful in clinical decision making, they need to be both reliable and valid. Substantial intrarater reliability of a hip abductor eccentric strength test has been reported ${ }^{5}$; however, the validity remains untested. For a test to be a valid measure of eccentric muscle strength, the peak force must occur during the lowering phase of the test. A previous descriptive study suggested that the peak force occurred during the lowering phase of a hip abductor eccentric strength test. ${ }^{4}$ However, this has not been statistically tested. Thus, the construct validity of hip abductor eccentric strength tests is unknown. Therefore, the purpose of this study was to determine intrarater reliability and construct validity of

The authors are with the Department of Physical Therapy and Rehabilitation Sciences, Drexel University, Philadelphia, PA, USA. Ebaugh is also with the Department of Health Sciences, Drexel University, Philadelphia, PA, USA. Brindle (brindle@drexel.edu) is corresponding author. a hip abductor eccentric strength test. We hypothesized that this test would have substantial intrarater reliability (intraclass correlation $[\mathrm{ICC}]>$.8). To determine construct validity, we hypothesized that the peak force would occur during the lowering phase of the test.

\section{Methods}

\section{Participants}

Prior to recruiting participants, this study protocol was approved by the Drexel University's Institutional Review Board. Healthy adults between 18 and 50 years old were recruited from the surrounding area. Twenty participants (18 women and 2 men) volunteered for this study (Table 1). All participants gave written informed consent prior to participating. Participants were pain free while walking, squatting, or moving their hips and had a body mass index less than $30 \mathrm{~kg} / \mathrm{m}^{2}$. A priori power analyses for ICC and a 1 -sample $t$ test were performed. For reliability, a minimum sample size of 19 was required to achieve an ICC of at least .7 with a target ICC of $.9 .{ }^{7}$ For the 1-sample $t$ test with an effect size of .8, alpha of .05 , and power of .8 , a minimum sample size of 15 was required. ${ }^{8}$ Therefore, at least 19 participants were needed to meet sample size requirements.

\section{Procedures}

Participants changed into shorts, and their height and weight were measured. The right limb was the test limb. The protocol followed the approach of Thorborg et al, ${ }^{5}$ with minor modifications intended to improve the reliability of the test. A line was drawn $5 \mathrm{~cm}$ proximal to the right lateral femoral epicondyle to standardize handheld dynamometer placement. The distance from this line to the ipsilateral greater trochanter was measured as the moment arm. During the test, participants lay on their left side on an examination 
table with their right hand holding the table's edge to stabilize their trunk (Figure 1). The right limb was fully extended and the left knee was flexed to $90^{\circ}$. To ensure proper positioning of the test limb, the tester moved the participant's right limb through maximum hip and knee flexion, then hip abduction followed by hip and knee extension. The limb was then lowered to $0^{\circ}$ hip abduction guided by an inclinometer. This provided a consistent frontal plane hip position across participants, which may not be achieved by placing the foot at a fixed height, as done in previous protocols. ${ }^{4-6}$ Transverse plane rotation in the test limb was minimized by ensuring that the lateral border of the foot was parallel with the table's surface. Standardized verbal instructions were given prior to each trial to "maintain this leg position using as much leg strength as you have until your leg reaches the table." First, a matching adduction force was applied to the test limb by the tester to elicit a maximum isometric contraction for approximately 2 seconds. Afterward, the tester gradually increased the adduction force until the test limb reached the table while contracting eccentrically. Three trials were recorded with at least 1 minute for rest after each test. Participants returned to the lab at least 1 week later and the protocol was repeated. The same tester collected all data during each visit for every participant.

The peak force and time to peak force during the test were recorded by the handheld dynamometer (Lafayette Instrument, Lafayette, IN). Limb motion was recorded at $100 \mathrm{~Hz}$ via an 8-camera motion capture system (Vicon, Oxford, UK), which tracked a retroreflective marker on the right tibial tuberosity. The handheld dynamometer made an audible signal when it began recording force to mark the start of each trial. After hearing the signal, the tester immediately remote-triggered the motion capture system to start recording.

Data were processed to determine the dependent variables for each participant. Peak force was multiplied by the moment arm to calculate torque, then divided by height and weight (\% BWh). The start of the lowering phase, when the limb began descending, was determined from the marker's vertical position using custom MATLAB software (MathWorks, Natick, MA). The start of the

\section{Table 1 Participant Demographic Information}

\begin{tabular}{lcc}
\hline & Mean (SD) & Range \\
\hline Age, y & $26(6)$ & $22-50$ \\
Height, m & $1.66(0.06)$ & $1.58-1.88$ \\
Weight, kg & $62.0(7.8)$ & $45.0-76.1$ \\
BMI, $\mathrm{kg} / \mathrm{m}^{2}$ & $22.4(2.0)$ & $18.1-24.8$ \\
\hline
\end{tabular}

Abbreviation: BMI, body mass index. lowering phase was identified by first connecting the initial and lowest vertical marker positions with a straight line. Then, the perpendicular distance from this line to the marker position was determined at each time point. The lowering phase began where the perpendicular distance was greatest (Figure 2). The start of the lowering phase was subtracted from the time to peak force to determine the peak force offset for each trial. A positive peak force offset indicated the peak force occurred during the lowering phase. Negative offsets indicated that the peak force occurred during the static phase.

\section{Statistical Analysis}

The normalized peak hip abduction torques from 3 trials were averaged for each participant. Intrarater reliability of the peak hip abduction torque was analyzed using $\mathrm{ICC}_{(3,3)}$ and interpreted using a standard index. ${ }^{9}$ The SEM and the minimal detectable difference (MDD) for $95 \%$ of a normal distribution were also determined. ${ }^{10}$

Negative peak force offsets were replaced with 0 for analysis. Peak force offsets were averaged for each participant. A 1-sample $t$ test was used to determine if the peak force offset during the test was greater than $0(P<.05)$. The tester's reaction time to an audible signal was determined using Inquisit 5.0 (Millisecond Software, LLC, Seattle, WA) to estimate the synchronization delay between the handheld dynamometer and motion capture system. The tester's reaction time to an audible signal averaged 0.25 seconds and ranged from 0.22 to 0.33 seconds over 5 trials.

\section{Results}

The mean peak hip abduction torque was $12.4(2.7) \% \mathrm{BWh}$ at the first visit and $13.2(2.5) \% \mathrm{BWh}$ at the second visit. The hip abductor eccentric strength test had substantial intrarater reliability, indicated by an ICC of .88 (95\% confidence interval, .65-.95). Additionally, the test had a SEM of $0.9 \%$ BWh and MDD of $2.5 \%$ BWh. Construct validity was established for the hip abductor eccentric strength test with an average peak force offset of 2.1 (0.6) seconds (range: $0.7-3.7 \mathrm{~s} ; P \leq .001$ ). Notably, the peak force offset was positive for all trials across all participants.

\section{Discussion}

The purpose of this study was to determine intrarater reliability and construct validity of the hip abductor eccentric strength test. We hypothesized that the test would have substantial intrarater

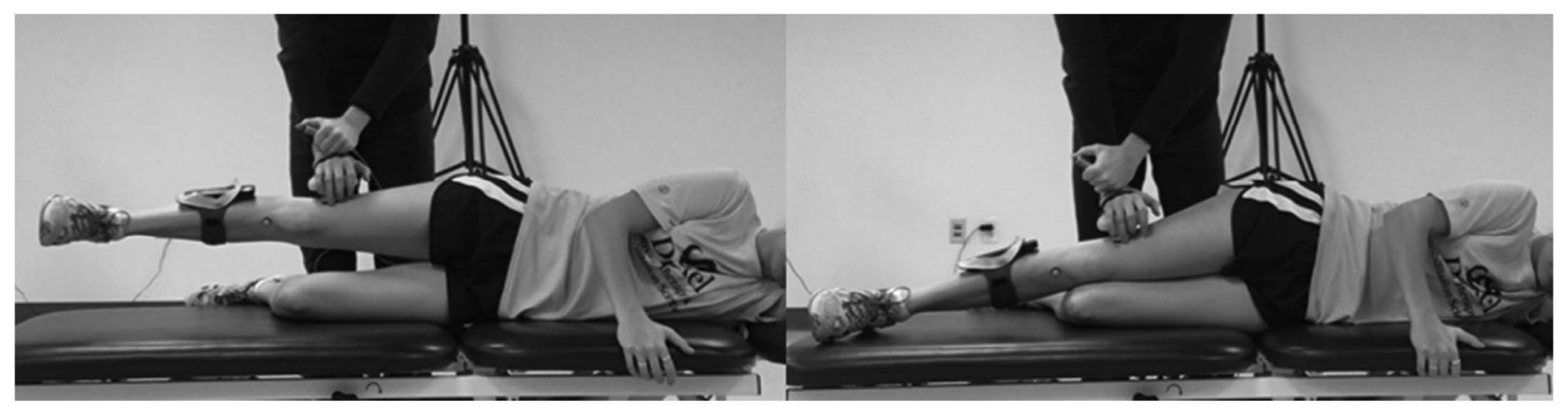

Figure 1 - Patient and tester positioning at the beginning and end position of the hip abductor eccentric strength test. 


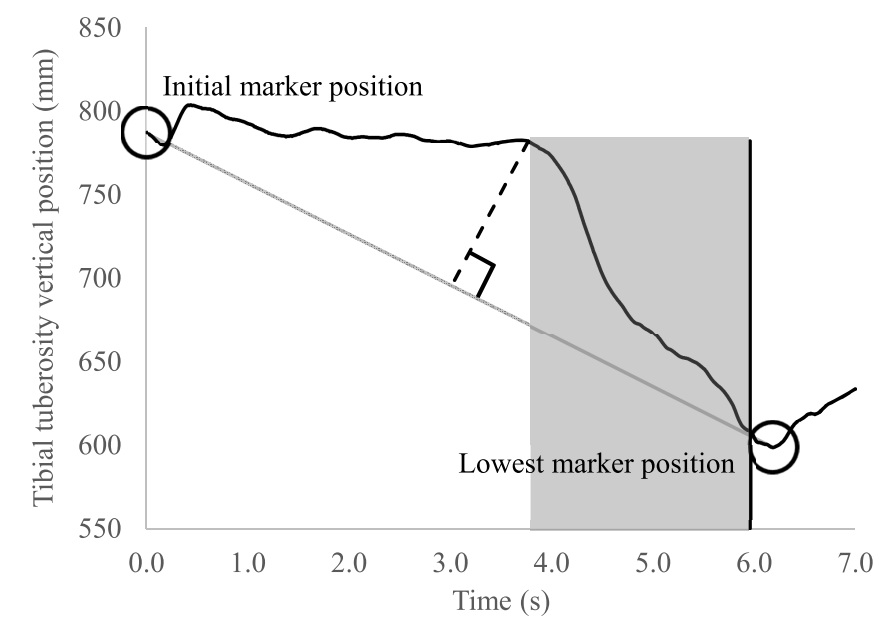

Figure 2 - Determination of the start of the lowering phase using the vertical position of the tibial tuberosity marker. Dashed line represents longest perpendicular distance from gray line to vertical position of tibial tuberosity, and shaded area represents peak force offset.

reliability and that the peak force would occur during the lowering phase. Both hypotheses were supported by the results. Objective strength tests must be reliable to be useful for clinical decision making. Our results are similar to the previously reported substantial intrarater reliability for another hip abductor eccentric strength test. ${ }^{5}$ Thus, we suggest using the MDD values from the present study with a general population. Objective measurements should quantify the intended construct to be valid and useful for clinical decision making. For a measurement of eccentric muscle strength to be valid, it must quantify muscle strength during an eccentric contraction. Maximum muscle force occurs during eccentric rather than isometric contraction. ${ }^{11}$ Therefore, peak hip abduction torque during a hip abductor eccentric strength test should occur during the lowering phase. A previous descriptive study suggested that this was the case. ${ }^{4}$ Our findings objectively confirmed those of the previous descriptive study and established construct validity for the hip abductor eccentric strength test.

We intentionally collected data in healthy participants to avoid the effects of injury or pain as potential cofounding variables. We acknowledge that the MDD may not apply to populations with specific pathologies. Thus, future studies should determine the MDD of this test in other populations. It was a limitation of this study that the handheld dynamometer and motion capture system were not digitally synchronized. However, the tester's reaction time indicated that the motion capture collection was delayed approximately 0.25 seconds relative to the dynamometer data. This delay between equipment was much smaller than the mean peak force offset of 2.0 seconds. Furthermore, the delay would have shortened the resulting peak force offset. Thus, if anything, the delay between equipment makes our findings more conservative.

In conclusion, the hip abductor eccentric strength test has substantial between-day intrarater reliability in healthy adults. We also established construct validity by demonstrating that peak force occurred during the lowering phase of the test. For clinical decision making, it is important to note that eccentric hip abductor muscle strength differences greater than $2.5 \% \mathrm{BWh}$ reflect a true difference in eccentric muscle strength between measurements. Differences less than $2.5 \% \mathrm{BWh}$ cannot be identified as a true difference because they fall within the error of measurement. For example, the MDD can be used in a clinical setting to determine if a true difference in eccentric hip abductor muscle strength occurred after an intervention. If the difference between the preintervention and postintervention peak hip abduction torque during the hip abductor eccentric strength test exceeded $2.5 \% \mathrm{BWh}$, then a true change occurred. The clinician can then determine whether the change is clinically meaningful. We recommend the hip abductor eccentric strength test using a handheld dynamometer for clinical use.

\section{References}

1. Boling MC, Padua DA, Alexander Creighton R. Concentric and eccentric torque of the hip musculature in individuals with and without patellofemoral pain. J Athl Train. 2009;44:7-13. PubMed ID: 19180213 doi:10.4085/1062-6050-44.1.7

2. Hewett TE, Myer GD, Ford KR, et al. Biomechanical measures of neuromuscular control and valgus loading of the knee predict anterior cruciate ligament injury risk in female athletes: a prospective study. Am J Sports Med. 2005;33:492-501. PubMed ID: 15722287 doi:10. 1177/0363546504269591

3. Robinson RL, Nee RJ. Analysis of hip strength in females seeking physical therapy treatment for unilateral patellofemoral pain syndrome. J Orthop Sports Phys Ther. 2007;37:232-238. PubMed ID: 17549951 doi:10.2519/jospt.2007.2439

4. Nicholas JA, Sapega A, Kraus H, Webb JN. Factors influencing manual muscle tests in physical therapy. J Bone Joint Surg Am. 1978;60:186-190. PubMed ID: 641082 doi:10.2106/00004623197860020-00008

5. Thorborg K, Couppe C, Petersen J, Magnusson SP, Holmich P. Eccentric hip adduction and abduction strength in elite soccer players and matched controls: a cross-sectional study. $\mathrm{Br}$ J Sports Med. 2011;45:10-13. PubMed ID: 19850576 doi:10.1136/bjsm.2009. 061762

6. Tyler TF, Nicholas SJ, Campbell RJ, McHugh MP. The association of hip strength and flexibility with the incidence of adductor muscle strains in professional ice hockey players. Am J Sports Med. 2001; 29:124-128. PubMed ID: 11292035 doi:10.1177/0363546501029 0020301

7. Walter SD, Eliasziw M, Donner A. Sample size and optimal designs for reliability studies. Stat Med. 1998;17:101-110. PubMed ID: 9463853 doi:10.1002/(SICI)1097-0258(19980115)17:1<101::AIDSIM727>3.0.CO;2-E

8. Faul F, Erdfelder E, Lang AG, Buchner A. G*Power 3: a flexible statistical power analysis program for the social, behavioral, and biomedical sciences. Behav Res Methods. 2007;39:175-191. PubMed ID: 17695343 doi:10.3758/BF03193146

9. Shrout PE. Measurement reliability and agreement in psychiatry. Stat Methods Med Res. 1998;7:301-317. PubMed ID: 9803527 doi:10. 1177/096228029800700306

10. Portney LG, Watkins MP. Foundations of Clinical Research: Applications to Practice. 3rd ed. Upper Saddle River, NJ: Pearson/ Prentice Hall; 2009.

11. Enoka RM. Eccentric contractions require unique activation strategies by the nervous system. J Appl Physiol. 1996;81:2339-2346. PubMed ID: 9018476 doi:10.1152/jappl.1996.81.6.2339 